\title{
Fracturas de cadera: satisfacción posquirúrgica al año en adultos mayores atendidos en Méderi-Hospital Universitario Mayor, Bogotá, D.C.*
}

\author{
Hip Fractures: Postsurgical Satisfaction a Year After in Older Adults Treated at Méderi-Hospital Universitario \\ Mayor, Bogotá, D.C.
}

Fraturas de quadril: Satisfação pós-cirúrgica no ano seguinte em idosos atendidos em Méderi-Hospital Universitário Mayor, Bogotá, D.C.

Iván Darío González MD 1,2, María Claudia Becerra MD², Juliana González MD2,5, Andrea Tatiana Campos $\mathrm{F}^{3,5}$, Jorge Barbosa Santibáñez MD ${ }^{4,5}$, José Ricardo Alvarado Sánchez MD, MPH2,5

Recibido: 17 de diciembre de 2015 - Aprobado: 29 de junio de 2016

Doi: https://dx.doi.org/10.12804/revsalud14.03.2016.08

Para citar este artículo: González ID, Becerra MC, González J, Campos AT, Barbosa-Santibáñez J, Alvarado R. Fracturas de cadera: satisfacción posquirúrgica al año en adultos mayores atendidos en Méderi-Hospital Universitario Mayor, Bogotá, D.C. Rev Cienc Salud. 2016;14(3):409-422. doi: https://dx.doi.org/10.12804/revsa-

lud14.03.2016.08

\section{Resumen}

Introducción: el envejecimiento poblacional mundial ha aumentado la incidencia de fracturas de cadera, este cambio genera un alto impacto económico, social y constituye un reto para la salud pública. Este estudio busca evaluar la satisfacción con el manejo posquirúrgico en adultos mayores con fracturas de cadera al año de la intervención y conocer los desenlaces en este tiempo. Materiales y métodos: estudio exploratorio, observacional, descriptivo de corte transversal, mediante encuesta telefónica en adultos de 65 o más años, un año después de intervención quirúrgica por fracturas de cadera en Méderi - Hospital Universitario Mayor, de Bogotá. Se describen variables evaluativas de satisfacción y atención en salud, así como otras relacionadas. Resultados: de 287 pacientes, 116 cumplen los criterios de inclusión y aceptan participar. El rango de edad oscila

\footnotetext{
* Estudio basado en la Tesis de Grado de Especialización en Epidemiología de Iván Darío González, MD y María Claudia Becerra, MD. Departamento de Salud Pública y Epidemiología, Escuela de Medicina y Facultad de Ciencias de la Salud, Universidad del Rosario. 
entre 65 y 99 años, media de 81,3 $\pm 8,17$. Las mujeres y los hombres representan, respectivamente, $76 \%$ y $24 \%$ de la muestra. Mortalidad anual $28 \%$, por género: $23 \%$ mujeres y $43 \%$ hombres. 29,3\% presenta reingresos hospitalarios, $50 \%$ recupera su habilidad para la marcha. $81 \%$ considera buena la atención intrahospitalaria por ortopedia. $64 \%$ no califica el servicio intrahospitalario por fisioterapia ya que no lo recibe. 70 \% expresa satisfacción con los servicios ambulatorios de ortopedia y rehabilitación. Conclusiones: estudio novedoso y único que da voz como sujeto activo al paciente para evaluar el sistema de salud, sugiere el grado de dolor como elemento trazador de calidad de vida y obliga a implementar programas y servicios para adultos mayores con fracturas de cadera.

Palabras clave: fracturas de cadera, adulto mayor, satisfacción posquirúrgica, capacidad funcional, rehabilitación.

\section{Abstract}

Introduction: World aging increases the incidence of hip fracture in adults over sixty years of age, pathology of economic and social burden being a challenge for public health. This study seeks to assess patient degree of satisfaction with health care services and assess outcomes. Materials and methods: Exploratory, observational, descriptive cross sectional study, with a telephone survey in patients 65 years or older, one year after hip fracture surgical intervention in MéderiHospital Universitario Mayor, Bogotá. Results: Of 287 patients 116 met the inclusion-exclusion criteria and accepted to participate. Age ranges between 65 and 99 years with a mean of 81,3 \pm 8,17 . Women and men represent respectively $76 \%$ and $24 \%$ of the sample. Mortality a year after is $28 \%$, gender: $23 \%$ women and $43 \%$ men. $29,3 \%$ of the patients reentered the hospital, $50 \%$ recovered their previous functional capacity. $81 \%$ express as good the inpatient care by orthopedic service. $64 \%$ does not grade inpatient care by rehabilitation services because it was not given. $70 \%$ are satisfied with the outpatient services of orthopedic and rehabilitation. Conclusions: This study represents a novel and unique approach as it gives voice to the patient allowing assessment of the health services. Suggests the degree of pain as a tracer of quality of life and brings upon compulsory attention to implement health services improvement programs for hip fractures in the elderly.

Keywords: Hip Fractures, Elderly, Satisfaction Assessment, Functional Recovery, Rehabilitation.

\section{Resumo}

Introdução: o envelhecimento populacional mundial tem aumentado a incidência de fraturas de quadril, mudanças que gera um alto impacto económico, social e constitui um desafio para a saúde pública. O estudo busca avaliar a satisfação com o manejo pós-cirúrgico em idosos com fraturas de quadril no ano posterior à intervenção e conhecer os desenlaces neste tempo. Materiais e métodos: estudo exploratório, observacional, descritivo, de corte transversal, mediante questionário por telefone em adultos de 65 ou mais anos, um ano após da intervenção cirúrgica por fraturas de quadril em Méderi - Hospital Universitário Mayor, de Bogotá D.C. Descrevem-se variáveis avaliativas de satisfação e atenção em saúde, assim como outras relacionadas. Resultados: de 287 
pacientes, 116 cumprem os critérios de inclusão e aceitam participar. O rango de idade oscila entre 65 e 99 anos, média de 81,3 $\pm 8,17$. As mulheres e os homens representam respetivamente $76 \%$ e $24 \%$ da amostra. Mortalidade anual 28\%, por gênero: $23 \%$ mulheres e 3\% homens. 29,3\% apresenta reingressos hospitalários, $50 \%$ recupera a sua habilidade para a marcha. $81 \%$ considera boa a atenção intra-hospitalar por ortopedia. $64 \%$ não qualifica o serviço intra-hospitalar por fisioterapia já que não o recebe. $70 \%$ expressa satisfação com os serviços ambulatórios de ortopedia e reabilitação. Conclusões: estudo novo e único que dá voz como sujeito ativo ao paciente para avaliar o sistema de saúde, sugere o grau de dor como elemento traçador de qualidade de vida e obriga a implementar programas e serviços para idosos com fraturas de quadril.

Palavras-chave: Fraturas de quadril, idoso, satisfação pós-cirúrgica, capacidade funcional, reabilitação.

\section{Introducción}

Las fracturas de cadera son una de las principales causas de morbimortalidad en las personas adultas mayores y representan un reto de salud pública en el mundo por su frecuencia y el alto costo económico y social que implican. En el año 1990 se informaron 1,3 millones de casos a nivel mundial, para el año 2050 se estima que lleguen a ocurrir entre 7 y 21 millones de casos $(1,2)$. En Colombia, se calcula que anualmente se presentan entre 8000 y 10000 fracturas de cadera solamente entre las mujeres; la osteoporosis es el principal factor de riesgo detectado en el grupo de mujeres mayores de 60 años $(3,4)$.

Existen varias clasificaciones para las fracturas de cadera, la más utilizada es la anatómica que las divide, según su ubicación con respecto a la cápsula del acetábulo, en intracapsulares y extracapsulares. La importancia clínica de esta clasificación radica en las diferencias terapéuticas y de pronóstico entre ellas $(5,6)$. La evidencia científica justifica el manejo quirúrgico oportuno de las fracturas de cadera dentro de las primeras 24-48 horas posteriores al diagnóstico para permitir la movilización temprana, disminuir el dolor, evitar complicaciones y prevenir el incremento en la mortalidad cuando el tiempo de espera para cirugía es mayor (7-9). Las infecciones del sitio operatorio, de vías urinarias, las ulceras por presión y la trombosis venosa profunda son las complicaciones más frecuentes observadas $(10,11)$.

La mortalidad en el mes posterior a las fracturas de cadera es de $5 \%$ a $10 \%$, y un $2 \%$ a 2,6\% de los pacientes mueren antes de recibir el manejo quirúrgico indicado. Al año de la cirugía, la mortalidad varía entre el $20 \%$ y el $31 \%(8,12-14)$. Una experiencia colombiana al respecto, publicada por Martínez Rondanelli en 2005, hace referencia a las condiciones médicas subyacentes, al estado general previo a las fracturas y a la presencia de comorbilidades, como los principales determinantes de la evolución del paciente e incremento de la mortalidad al año de la cirugía (8).

El manejo del adulto mayor con fracturas de cadera, dada su complejidad, requiere un equipo multidisciplinario que incluya cuidadores, médicos especialistas y rehabilitación para lograr recuperar el nivel de independencia $(14,15)$. 
En Colombia, actualmente, se experimenta un cambio en la pirámide poblacional dado por el incremento de adultos mayores, los cuales representan el 10,5\% de la población total $(16,17)$. Se desconoce la incidencia real de fracturas de cadera en Colombia y en la ciudad de Bogotá; por lo tanto, es fundamental tener un marco comparativo y conocer ciertos datos relevantes de la población de adultos mayores de la ciudad. Actualmente, esta población es de 817800 individuos y representa el 10,7\% de la población total de la ciudad (7674366) (18, 19). Se presenta discapacidad permanente en $53 \%$ de los adultos mayores y alteraciones permanentes de cadera y pies en $25,3 \%$ de ellos, $52,4 \%$ de los adultos mayores de la ciudad, de acuerdo con el índice de fragilidad, se clasifican como prefrágiles y 28,5\% de ellos presenta al menos una caída al año (19). Estos cambios se traducen en una mayor prevalencia de enfermedades crónicas como las fracturas de cadera y la discapacidad permanente.

Para enfrentar este reto demográfico y económico actual del país, se cuenta con un sistema de salud cuyos postulados se dirigen hacia la atención integral en salud, pero cuyas características administrativas, por medio de las Empresas Promotoras de Salud (EPS), dificultan el modelo integral de atención en salud en una única Institución Prestadora de Servicios (IPS) (20). El manejo es fragmentado, y dicha condición no exceptúa a los pacientes adultos mayores con fracturas de cadera; manejo que afecta el control y seguimiento adecuados fundamentales para su recuperación (21).

La magnitud del problema descrito y en especial la realidad del sistema de salud en Colombia motivaron la realización del estudio cuyo objetivo general es el de evaluar la satisfacción de los adultos mayores con el manejo hospitalario y ambulatorio de los servicios de ortopedia y fisioterapia durante el proceso enfermedad - salud un año después de haber sido intervenidos quirúrgicamente por fracturas de cadera en una IPs de alta complejidad, Méderi - Hospital Universitario Mayor (HUM) de la ciudad de Bogotá.

Los objetivos específicos del estudio buscan caracterizar demográficamente la población, conocer los desenlaces en el tiempo, en términos de mortalidad, tiempo de espera para la cirugía, reingresos hospitalarios y motivo de ellos, grado de dolor y capacidad funcional.

Se espera que los resultados obtenidos permitan mejorar los procesos de atención, incentiven la realización de estudios de incidencia y prevalencia que sirvan de base para estudios longitudinales y de intervención de mayor complejidad, y generen la necesidad imperiosa de implementar políticas de manejo que en forma integral le permitan al adulto mayor incorporarse en las mejores condiciones y en el menor tiempo posible a su actividad cotidiana previa.

\section{Materiales y métodos}

Se realiza un estudio exploratorio, observacional, descriptivo, de corte transversal durante un año, comprendido entre el 1 julio de 2012 y el 30 de junio de 2013, mediante una encuesta telefónica estructurada en pacientes con 65 años o más o sus cuidadores, un año después de la intervención quirúrgica por fracturas de cadera en Méderi - Hum de la ciudad de Bogotá.

Los criterios de inclusión son: (i) Pacientes de 65 o más años, hombres y mujeres, con intervención quirúrgica por fracturas de cadera en el periodo descrito. (ii) Primer episodio de fracturas de cadera. (iii) Contar con datos de contacto telefónico. Los criterios de exclusión son: (i) Imposibilidad para contactar al paciente o su cuidador. (ii) Negación del paciente o su cuidador a participar en la encuesta. 
Tabla 1. Cuestionario Estandarizado - Encuesta Telefónica

\begin{tabular}{|c|}
\hline Preguntas para determinar reingresos hospitalarios durante el año posterior a la cirugía \\
\hline $\begin{array}{l}\text { 1. ¿El paciente tuvo que reingresar nuevamente al hospital? Sí/NO } \\
\text { 2. ¿Cuál fue el motivo? }\end{array}$ \\
\hline $\begin{array}{c}\text { Preguntas para evaluar grado de dolor y capacidad funcional previos a las fracturas de cadera. Las mismas preguntas } \\
\text { para evaluar } 1 \text { año después de la cirugía }\end{array}$ \\
\hline $\begin{array}{l}\text { 3. ¿El grado de dolor del paciente fue leve, moderado o severo? } \\
\text { 4. ¿El paciente caminaba antes de la cirugía? SÍ/NO } \\
\text { 5. ¿El paciente necesitaba ayuda? SÍ/NO } \\
\text { 6. ¿El paciente podía caminar dentro y fuera de la casa? Sí/NO } \\
\text { 7. Si el paciente caminaba únicamente dentro de la casa, ¿lo hacía sin ayuda o supervisión? } \\
\text { 8. ¿Con ayuda o supervisión? } \\
\text { 9. ¿El paciente podía trasladarse desde la silla a la cama sin ayuda, con mínima ayuda, con gran ayuda, era incapaz de } \\
\text { permanecer sentado? } \\
\text { 10. ¿El paciente es capaz de subir y bajar un piso sin la ayuda ni supervisión de otra persona? } \\
\text { 11. ¿Precisa ayuda o supervisión? } \\
\text { 12. ¿Es Incapaz de subir o bajar escalones? }\end{array}$ \\
\hline $\begin{array}{c}\text { Preguntas para evaluar satisfacción del paciente con manejo hospitalario y ambulatorio de los servicios de ortopedia y } \\
\text { fisioterapia. Pregunta abierta N. }{ }^{\circ} 21 .\end{array}$ \\
\hline $\begin{array}{l}\text { 13. ¿Recibió manejo por fisioterapia durante la hospitalización? SÍ/NO } \\
\text { 14. ¿Podría seleccionar el grado de satisfacción con este servicio? SÍ/NO } \\
\text { 15. ¿El servicio de fisioterapia fue bueno, regular o malo? } \\
\text { 16. ¿Podría seleccionar el grado de satisfacción con el servicio de ortopedia? Sí/NO } \\
\text { 17. ¿El servicio de ortopedia fue bueno, regular o malo? } \\
\text { 18.. ¿Tuvo control por consulta externa con ortopedia después de la cirugía? SÍ/NO } \\
\text { 19. ¿Tuvo control por consulta externa con fisioterapia después de la cirugía? SÍ/NO } \\
\text { 20. ¿Está usted satisfecho con el seguimiento que recibió después de haber salido del hospital? Sí/NO } \\
\text { 21. ¿Qué recomendaciones o sugerencias deja usted para mejorar el servicio a futuros pacientes con fracturas de cadera? }\end{array}$ \\
\hline
\end{tabular}

Los datos de contacto se obtienen por medio del departamento de estadística del HuM, se combinan el grupo de diagnósticos relacionados con fracturas de cadera, el grupo etáreo, la fecha de ingreso y el código único de procedimientos diagnósticos en salud (cups) de cirugías de cadera. Se realiza una revisión individual de historias clínicas para asegurar criterios de inclusión y exclusión. Se construye encuesta telefónica estandarizada que se aprueba por el comité de expertos del Hum (tabla 1).

Se realizan tres series de llamadas por el mismo profesional en medicina hasta obtener respuesta. La primera pregunta hace referencia al asentimiento verbal y voluntario de participación y su grabación bajo los compromisos de confidencialidad y uso de datos con fines científicos y de mejoramiento de la calidad de los servicios. El respondiente tiene la libertad de aceptar o no participar en la encuesta y de no responder aquellas preguntas que considera inapropiadas o que no desea contestar. Si el respondiente acepta, se realizan las preguntas restantes del cuestionario (tabla 1 ).

Los posibles sesgos de medición, instrumento y fatiga se minimizan al realizar prueba piloto, encuesta con cuestionario estandarizado de respuesta única con una duración menor de 20 minutos. El sesgo de memoria es de difícil control, dados los posibles desenlaces vitales.

Se definen las variables, según se describen en la tabla 2. La información se exporta a Excel ${ }^{\circledR}$ y spss Statistic para su análisis descriptivo, se realiza una caracterización de la 
Tabla 2. Definición de las variables utilizadas

\begin{tabular}{|c|c|}
\hline Nombre de la variable & Definición conceptual \\
\hline Nombre de cups & $\begin{array}{l}\text { Nombre del código único de procedimientos relacionados con cirugías por } \\
\text { fracturas de cadera }\end{array}$ \\
\hline Participa estudio & No desea participar / Acepta participar en el estudio \\
\hline Participante de la encuesta & Paciente / Cuidador Familiar / Cuidador sin parentesco \\
\hline Género & Femenino / Masculino \\
\hline Edad al ingreso & Años cumplidos al momento de la fractura \\
\hline Mortalidad & $\begin{array}{l}\text { Falleció en el año posterior a la cirugía. No fallecido / Falleció / No sabe / } \\
\text { No responde }\end{array}$ \\
\hline Tiempo de espera para cirugía & $\begin{array}{l}\text { Tiempo que transcurre desde fecha ingreso por fracturas cadera hasta fecha } \\
\text { de cirugía. Rangos } 0 \text { - } 3 \text { días / 4-6 días / } 7 \text { o más días }\end{array}$ \\
\hline Reingresos & $\begin{array}{l}\text { Reingreso a hospitalización durante el año posterior al tratamiento qui- } \\
\text { rúrgico de las fracturas de cadera. No reingresa / Reingresa / No sabe / No } \\
\text { responde }\end{array}$ \\
\hline Motivo primer reingreso & $\begin{array}{l}\text { Enfermedad pulmonar / Infección Sitio operatorio / Trastornos osteomus- } \\
\text { culares / Enfermedad Cardiovascular /Trombosis venosa profunda* }\end{array}$ \\
\hline Motivo segundo reingreso & $\begin{array}{l}\text { Enfermedad pulmonar / Infección Sitio operatorio / Trastornos osteomus- } \\
\text { culares / Enfermedad Cardiovascular /Trombosis venosa profunda* }\end{array}$ \\
\hline Grado de dolor & Leve / Moderado / Severo / Ausencia de dolor \\
\hline Capacidad Funcional & $\begin{array}{l}\text { Nivel desempeño marcha, realizar actividades instrumentales vida diaria } \\
\text { antes de las fracturas, un año después de la cirugía. } \\
\text { Respuestas a las preguntas } \text { N. }^{\circ} 4 \text { a N } .^{\circ} 12 \text { de encuesta telefónica (tabla 1) }\end{array}$ \\
\hline $\begin{array}{l}\text { Satisfacción paciente con servicios hos- } \\
\text { pitalarios ortopedia / fisioterapia }\end{array}$ & Respuestas a las preguntas $\mathrm{N}^{\circ} 13$ a N. ${ }^{\circ} 17$ de encuesta telefónica (tabla 1 ) \\
\hline $\begin{array}{l}\text { Satisfacción paciente con servicios am- } \\
\text { bulatorios ortopedia / fisioterapia. }\end{array}$ & Respuestas a las preguntas $\mathrm{N} .^{\circ} 18$ a N $^{\circ} 20$ de encuesta telefónica (tabla 1 ) \\
\hline Comentarios y/o sugerencias & $\begin{array}{l}\text { Comentarios y/o sugerencias pacientes sobre servicios intra y extrahospita- } \\
\text { larios Respuestas a la pregunta } \mathrm{N}^{\circ} 21 \text { de la encuesta telefónica (tabla 1) }\end{array}$ \\
\hline
\end{tabular}

*Se utilizan 22 categorías diagnósticas de las cuales, se incluyen en la definición conceptual las cinco más frecuentes.

población estudiada, se comparan los datos de funcionalidad y dolor antes de la fractura y un año después de la cirugía. Se utilizan medidas de tendencia central, media y desviación estándar para las variables cuantitativas y frecuencias, porcentajes y gráficas de barra para las variables cualitativas.

Se presenta en las Sesiones del Comité Técnico de Investigaciones de Méderi-HuM, se aprueba y califica según Resolución 008430 como investigación sin riesgo, dado que no se realiza ninguna intervención o modificación de las variables biológicas, fisiológicas, sicológicas o sociales de los individuos que participan en el estudio.

\section{Resultados}

Selección y caracterización demográfica de la población: durante un año, 279 pacientes ingresaron por fracturas de cadera a la institución y representaron la población de estudio, 125 no accedieron a responder la encuesta y 9 no cumplieron los criterios de inclusión. La muestra se conformó por 116 pacientes que cumplie- 
ron con los criterios de inclusión-exclusión. El respondiente de la encuesta fue un cuidador familiar en 84 casos, el paciente en 27 , y un cuidador sin parentesco en 5 casos.

El rango de edad osciló entre 65 y 99 años, con una media de 81,3 años (DS: 8,17). Con respecto al género, $76 \%$ fueron mujeres y $24 \%$ hombres.

La mortalidad informada por el respondiente en el primer año después de la cirugía fue $28 \%$, fallecieron 32 de 116 pacientes. Por género, la mortalidad masculina $43 \%$, y la femenina $23 \%$.

Tiempo espera para cirugía: en $52 \%$ de los casos fue de 1 a 3 días, en $40 \%$ de 4 a 6 días y en $8 \%$, de 7 y más días.

Reingresos hospitalarios: En el año posterior a la cirugía, $60 \%$ de los pacientes no presentó reingresos hospitalarios, 29,3 \% reingresó y en $10 \%$ de los casos no se obtuvo información clara al respecto. Un segundo reingreso se presentó en $9 \%$ de los pacientes. Los motivos más frecuentes informados fueron: Enfermedades pulmonares, infecciones del sitio operatorio, trastornos osteomusculares, enfermedades cardiovasculares y trombosis venosa profunda.

Grado de dolor: la figura 1 ilustra el grado de dolor en fracturas de cadera previo a la cirugía. 70 pacientes $(60,3 \%)$ refirieron dolor severo, 21 (18\%), moderado y $17(14,6 \%)$, leve. Un año después de la cirugía, 11 (9,4\%) refirió dolor severo, 34 (29,3\%), moderado y 63 $(54,3 \%)$, leve. Ninguno de los pacientes refirió ausencia de dolor.

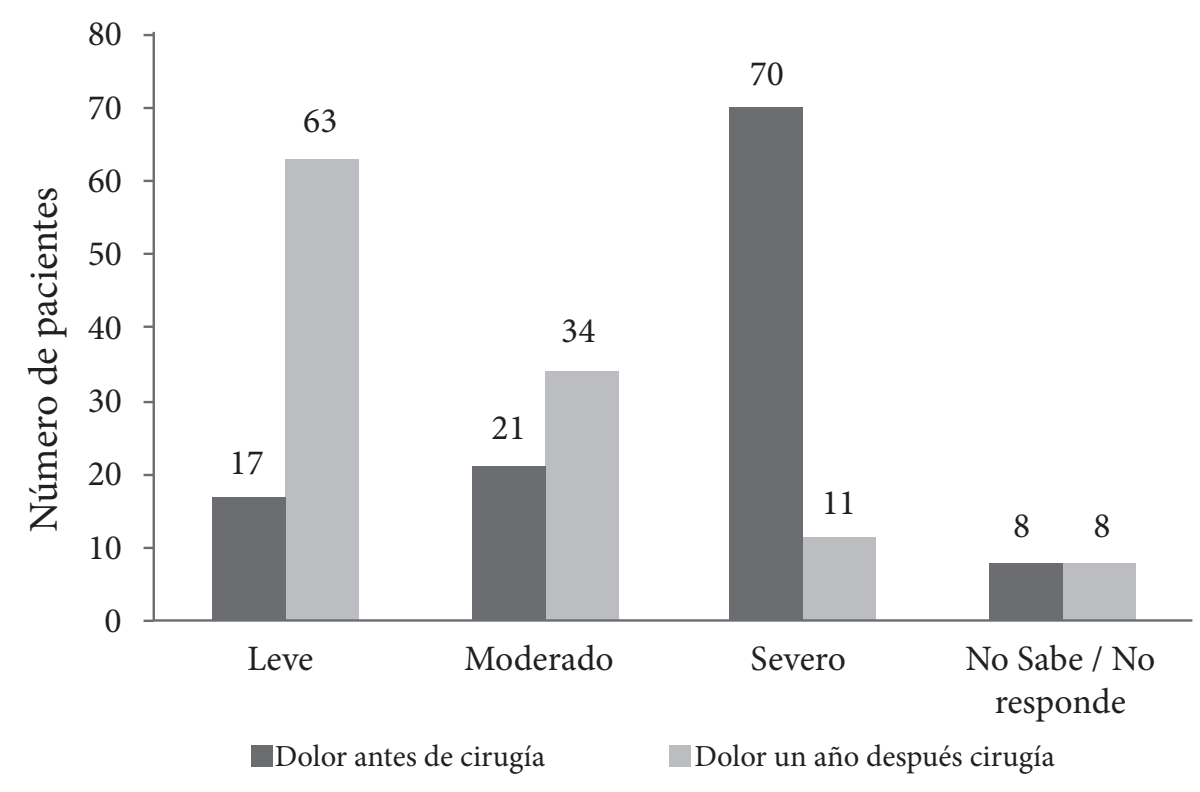

Figura 1. Fracturas de cadera y grado de dolor previo a la cirugía y un año posterior a la intervención quirúrgica $(n=116)$ 


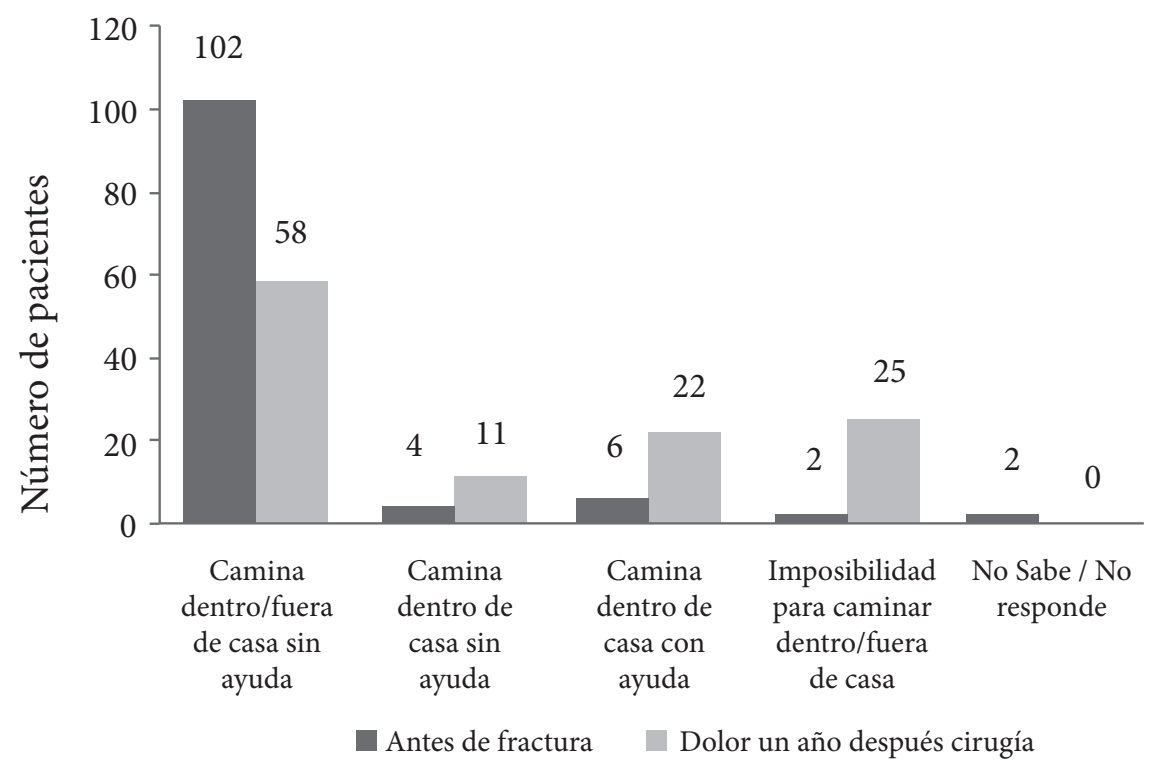

Figura 2. Desempeño en la marcha, previo a fracturas de cadera y un año después del procedimiento quirúrgico $(n=116)$

Capacidad funcional: en la figura 2 se observa previo a las fracturas de cadera que 102 pacientes $(88 \%$ ) podía caminar dentro y fuera de casa sin ayuda, al año del procedimiento 58 (50\%) pudo realizar esta misma actividad. Se incrementó de $12 \%$ a $50 \%$ el número de casos en que la marcha se limitó a la casa, con o sin ayuda, o hubo imposibilidad completa para ella.

Los parámetros de la actividad instrumental de la vida diaria evaluados fueron la capacidad de traslado silla/cama y la capacidad para subir/ bajar un piso. Previo a las fracturas, $87 \%$ y $75 \%$, respectivamente, podían realizar estas actividades sin ayuda. Un año después de la cirugía, disminuyó el número de pacientes que podía realizar las actividades sin ayuda a 51,7\% y a $26,7 \%$, respectivamente. Aumentó la necesidad de ayuda para las dos evaluaciones y en $16 \%$ de los casos se imposibilitó la realización de estas actividades.

Satisfacción con la atención hospitalaria por ortopedia - fisioterapia: la figura 3 ilustra la evaluación del paciente sobre la atención prestada por los servicios de ortopedia y fisioterapia durante su hospitalización. $81 \%$ se sintió satisfecho con la atención prestada por el servicio de ortopedia y la calificó como buena, $12 \%$, como regular y mala, y $7 \%$ prefirió no calificarla. $64 \%$ de los pacientes no evaluó el servicio de fisioterapia durante la hospitalización, 6 \% lo consideró regular y malo y 30 \% lo evaluó como bueno. La razón de estos hallazgos obedeció a la falta de atención por fisioterapia durante la hospitalización en más de la mitad de los casos.

Evaluación satisfacción control ambulatorio por ortopedia - fisioterapia: de los 116 pacientes del estudio, 94 refirieron haber sido valorados ambulatoriamente por ortopedia (81\%) y 86 por fisioterapia (74\%). 12 no refirieron atención ambulatoria por ortopedia y 20 no refirieron haber sido atendidos por fisioterapia, 10 pacientes no respondieron esta pregunta. Los resultados los refleja la figura 


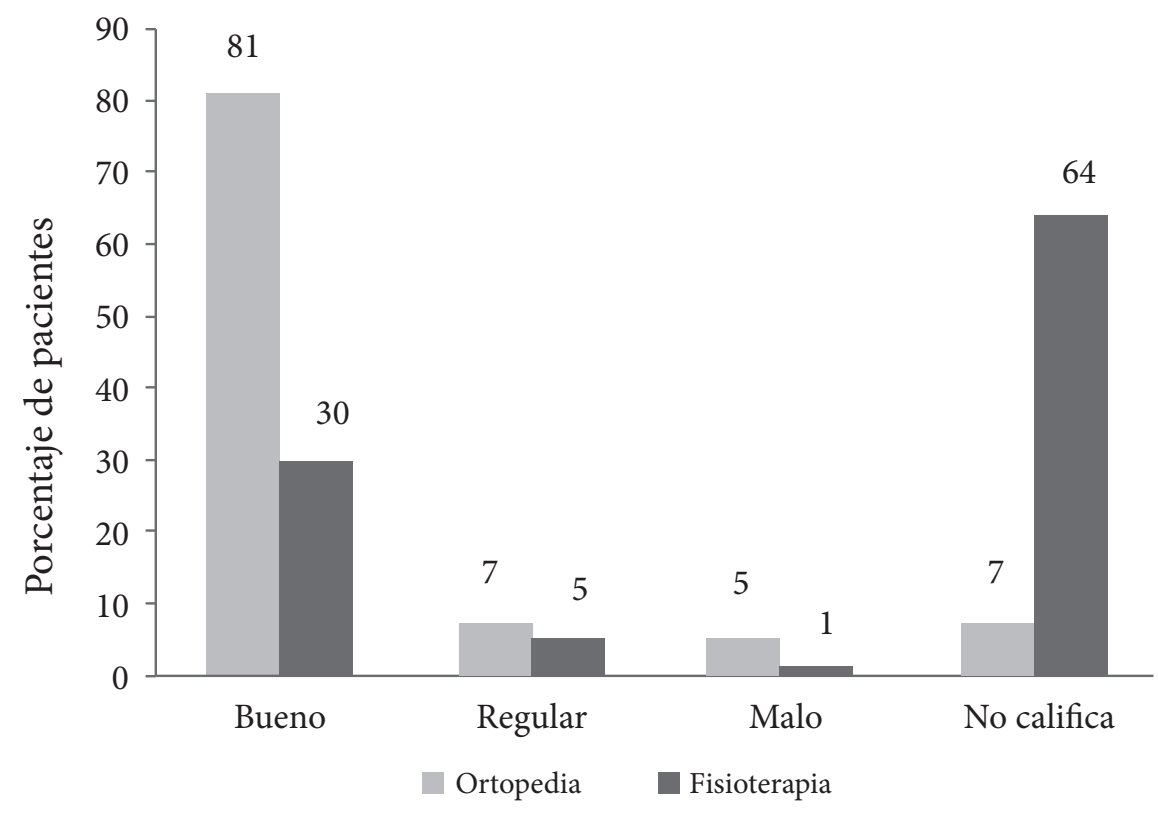

Figura 3. Evaluación de la satisfacción del paciente con la atención por ortopedia y fisioterapia durante la hospitalización por tratamiento quirúrgico de fracturas de cadera $(n=116)$

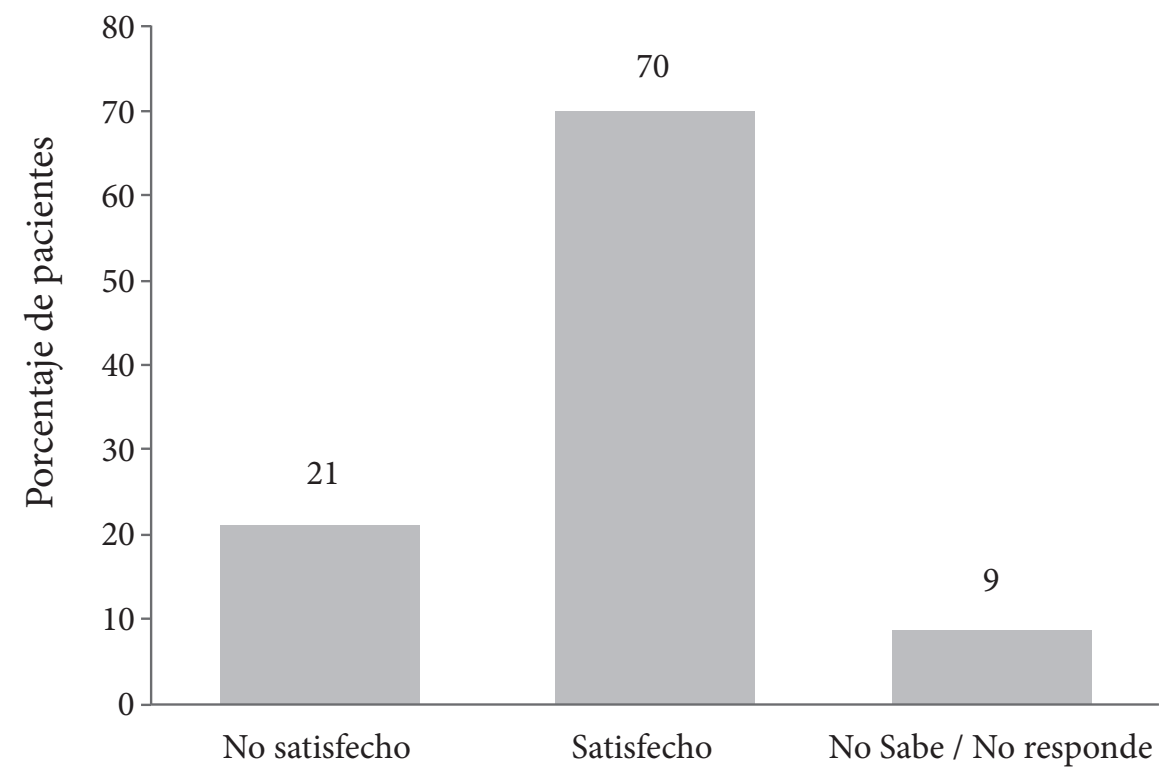

Figura 4. Evaluación de satisfacción del paciente con el control ambulatorio de ortopedia y fisioterapia a lo largo de un año posterior al tratamiento quirúrgico de fracturas de cadera $(n=116)$ 
Tabla 3. Necesidades y Expectativas del paciente de acuerdo con el grupo responsable

\begin{tabular}{l}
\hline \multicolumn{1}{c}{ Empresa Promotora de Salud (EPS) } \\
\hline Que se garantice el ACCESO a los servicios de salud y a consultas con especialista \\
AGILIZAR las citas de control con el especialista \\
Que haya CONTINUIDAD con el especialista en las consultas del postoperatorio \\
AUTORIZAR el acceso a rehabilitación hospitalaria \\
Que se AUTORICEN terapias de rehabilitación ambulatoria en forma domiciliaria \\
Facilitar el acceso o sUMINISTRAR las ayudas necesarias para la marcha del paciente \\
Que se PRIORICE el apoyo con los pacientes que sufren fracturas de cadera y además si son discapacitados o padecen \\
enfermedades terminales \\
\hline IPS - Méderi - Hospital Universitario Mayor (HUM) \\
$\quad$ Profesionales de la salud \\
\hline Que se brinde INFORMACIÓN sobre el diagnóstico tanto al paciente como a su familia \\
Que se EXPLIQUE claramente a los cuidadores los elementos requeridos y las adecuaciones necesarias en el hogar \\
Que muestren INTERÉS y sepan ESCUCHAR a cada uno de los pacientes \\
Que NO DEMOREN el tratamiento quirúrgico \\
Que la rehabilitación sea constanTE \\
Que EVITEN los eventos adversos en salud que pudieran lesionar y agravar al paciente \\
Que LLAMEN al paciente para conocer su evolución, no solo con fines investigativos \\
\hline
\end{tabular}

4 con el grado de satisfacción que expresó el paciente con respecto a los servicios evaluados durante el periodo ambulatorio.

Comentarios/sugerencias referidos por los entrevistados: la pregunta final con respuesta abierta de la encuesta: $i$ Qué recomendaciones o sugerencias deja usted para mejorar el servicio a futuros pacientes con fracturas de cadera? fue respondida por 38 de 116 pacientes o sus cuidadores, las respuestas representaron necesidades y expectativas de los pacientes y fueron categorizadas de acuerdo con el grupo responsable de cada proceso como se ilustra en la tabla 3.

\section{Discusión}

Las fracturas de cadera son un problema cada vez más común en la población de adultos mayores y Colombia no es ajena a esta problemática (17-19). En el país, los estudios poblacionales sobre vejez de alcance nacional son pocos, aunque el marco normativo referente a este tema se haya reformado varias veces en los últimos años como respuesta al envejecimiento contundente de la población (22). En un futuro próximo, el envejecimiento demográfico y el aumento en el volumen de la población adulta mayor del país será un problema sobresaliente, por lo que debe ser priorizado para su planificación económica y social $(18,19)$. En respuesta a ello, cada vez son más frecuentes en el país los estudios que revisan los sistemas y modelos de salud y su incidencia en la prestación de los servicios de salud, así como aquellos que utilizan la percepción como herramienta para el mejoramiento continuo en los servicios de salud (22-26). Sin embargo, para enfrentar el reto que las enfermedades crónicas como las fracturas de cadera representan para la salud pública se debe tener un mejor conocimiento de ellas, y en Colombia y en la ciudad de Bogotá se desconoce la incidencia real de fracturas de cadera, son escasos los estudios existentes y la mayoría hacen referencia a las características demográficas, mortalidad, complicaciones y recuperación funcional. No se encontró en la literatura colombiana o hispanoamericana revisada un estudio que evaluara la satisfacción del adulto mayor intervenido por fractura 
de cadera con los servicios involucrados en su proceso enfermedad-salud. El presente análisis no constituye un estudio de percepción, pero sí uno exploratorio y novedoso que brinda datos de la magnitud del problema del manejo intra- y extra- hospitalario de las fracturas de cadera y del sistema de salud en un hospital universitario de alta complejidad en Bogotá. En este modelo de investigación, el paciente, al convertirse en participante activo del estudio, manifiesta problemas reales de los servicios evaluados que pueden ser poco alentadores para aquellos involucrados, y generar insatisfacción o falta de motivación, pero cuyo objetivo primordial es el de interesarlos en la solución y el mejoramiento urgente de los problemas y necesidades. Los pacientes expresaron claramente su insatisfacción con el servicio de fisioterapia durante su hospitalización, $64 \%$ no calificó el servicio ya que no recibió atención; 81 \% manifestó satisfacción con el servicio de ortopedia durante su hospitalización y evalúo como buena la atención. En el control ambulatorio a lo largo del año posterior a la intervención quirúrgica, 70 \% de los pacientes se sintió satisfecho con la atención por parte de los servicios de ortopedia y fisioterapia; sin embargo, 30 \% expresó insatisfacción o no calificó los servicios. Al conocer las características ya mencionadas del sistema de salud colombiano, se esperaba encontrar una evaluación inversa, con mejores resultados durante la hospitalización que durante la fase ambulatoria, es por ello prioritario que el hospital y los servicios evaluados conozcan, analicen e intervengan el problema, encuentren soluciones y las ejecuten en la mayor brevedad posible.

Los comentarios y/o sugerencias en respuesta abierta a la última pregunta de la encuesta ¿Qué recomendaciones o sugerencias deja usted para mejorar el servicio a futuros pacientes con fracturas de cadera? fueron aportados por 38 pacientes y reflejaron unos, los problemas que genera la disociación existente entre aseguramiento y prestación de servicios de salud que pueden ser la causa de la fragmentación del sistema que origine los problemas de integralidad y continuidad en la atención del paciente (21). Otros, que hacen referencia al personal hospitalario, pues aseguraron que se debe generar en este la cultura del contexto social en la estructuración del desarrollo humano y considerar los pacientes y especialmente los adultos mayores, como seres que integran un núcleo familiar y social que va a ser su futura red de apoyo, fundamental en el proceso de recuperación en las cirugías por fracturas de cadera. Se debe entender el componente emocional del paciente y su familia ante un evento tan serio y doloroso y brindar toda la información que requiera el paciente y su red de apoyo para permitir al adulto mayor una pronta y mejor recuperación, lograr un envejecimiento activo, entendido este como la capacidad de influir en el curso de su vida con autonomía y desarrollo de posibilidades.

El componente descriptivo del estudio mostró datos acordes con los informados en la literatura en cuanto al promedio de edad $(81,3$ años), el género (76\% mujeres, $24 \%$ hombres), la mortalidad (28\%) y la mortalidad por género ( $23 \%$ mujeres, $43 \%$ hombres), un año después de la cirugía por fracturas de cadera (3, $8,9,13,14)$. Se destaca el tiempo de espera para la cirugía de 1 a 3 días en $52 \%$ de los casos, que comparado con los informados en la literatura colombiana revisada, es un tiempo corto si se considera aquellos mayores a 6 días y de 7,8 días $(8,9)$.

Entre los factores predictivos de buen pronóstico para la recuperación funcional de los pacientes, cabe destacar, entre otros, la capacidad para la marcha, no necesitar de ayuda para deambular, y caminar dentro/fuera de casa 
sin ayuda previo a las fracturas de cadera (15). La evaluación funcional comparativa de estos parámetros en el estudio mostró, al año de la cirugía, una recuperación de la capacidad para la marcha sin ayuda en $50 \%$ de los pacientes, dato que se encuentra dentro de los rangos informados en la literatura $(8,15,27)$. La calidad de vida del paciente al año de la cirugía por fracturas de cadera se va a ver afectada por la presencia de dolor persistente moderado o severo (28). En el estudio, un año después de la cirugía, el dolor fue leve en $54,3 \%$ de los casos, moderado en $29,3 \%$, y severo en $9,4 \%$, hallazgos que ameritan desarrollar estrategias de control del dolor en adultos mayores intervenidos quirúrgicamente por fracturas de cadera.

El estudio presenta las debilidades propias de un estudio descriptivo como son los registros incompletos en la historia clínica de complicaciones y principalmente de los tipos de fracturas de cadera intra o extracapsulares, dadas las diferencias etiológicas, terapéuticas y pronósticas para cada una de ellas. La principal fortaleza del estudio es la de entregar una visión novedosa en el ámbito de las fracturas de cadera, relacionada con la percepción de los pacientes o sus cuidadores, que son utilizados en forma central y activa en el desarrollo del estudio.

\section{Conclusiones:}

- El estudio en un hospital universitario constituye un aporte novedoso y único al dar voz y carácter de sujetos activos a los pacientes para evaluar el sistema y los servicios de salud.

- Pone de manifiesto cómo los pacientes perciben claramente las deficiencias en el manejo postoperatorio de las fracturas de cadera relacionadas, fundamentalmente, con la fase hospitalaria y la implementación de los sistemas de rehabilitación motora.
- Evidencia la necesidad de realizar en la evaluación inicial del adulto mayor con fracturas de cadera, la descripción objetiva del nivel de funcionalidad previo y urge que estos y otros datos se consignen clara y completamente en las historias clínicas de los pacientes.

- Permite sugerir la persistencia del dolor con una entidad trazadora de calidad de vida en los adultos mayores intervenidos por fracturas de cadera.

- Se convierte en una herramienta útil para el mejoramiento continuo y práctico de los procesos de salud, su socialización fomentará la participación de las directivas y la academia en la integración de nuevos modelos de servicios de salud que permitan garantizar la accesibilidad, la integralidad y la continuidad en la atención.

- Busca transformar a los profesionales de la salud en facilitadores de procesos científicos y sociales, cuyo objetivo sea lograr un adulto mayor autónomo y crear en el ámbito hospitalario, en los pacientes y en sus familias, un imaginario positivo de la vejez.

\section{Agradecimientos}

Los autores agradecen especialmente la colaboración del Centro de Investigaciones de Méderi(CIMED), del Señor Alex Arroyo, Departamento de Estadística del HuM, de los Doctores Francisco González, Jefe de Medicina Interna, MéderiHuM, Guillermo Rueda, Departamento de Ortopedia de Méderi-HUM, Álvaro López, Psiconeurólogo, Méderi-Hum, Martha Ramírez, Jefe de Atención al Usuario, Méderi-Hum, Doctora Patricia Bonilla, Patóloga y de los profesionales en medicina Doctores Marlyn Campo Arias, Alejandra Pereira Vidal, Estephanía Luna Pisciotti, Daniel González Sánchez, Steven Rojas Guerrero, Pablo Bermúdez por su esfuerzo y colaboración en las diferentes etapas del proyecto. 
Descargos de responsabilidad Los autores del presente artículo declaran no tener ningún tipo de conflicto de interés eco- nómico o científico en la elaboración del estudio, recolección de datos o análisis de estos. La financiación se realizó con recursos propios.

\section{Referencias}

1. Kannus P, Parkkari J, Sievänen H, Heinonen A, Vuori I, Járvinen M. Epidemiology of hip fractures. Bone 1996;18:57-63.

2. Gullbertg B, Johnell O, Kanis J. Worldwide projections for hip fracture. Osteoporos Int 1997;7:407-13.

3. International Osteoporosis Foundation. Facts and statistics. International Osteoporosis Foundation.

4. González LA, Vásquez GM, Molina JF. Epidemiología de la osteoporosis. Rev. Colomb. Reumatol 2009;16:61-75.

5. Minnesota Evidence-based Practice Center, Butler M, Forte M, Kane RL, Joglekar S, Duval SJ, et al. Treatment of common hip fractures. Evidence Report/Technology Assessment No. 184. AHRQ Publication N. ${ }^{\circ}$ 09-E013. Rockville, MD: Agency for Healthcare Research and Quality; 2009.

6. Muñoz S, Lavaderos J, Vilches L, Delgado M, Cárcamo K, Passalaqua S, et al. Fractura de cadera. Cuad. Cir 2008;22:73-81.

7. Ren-shi MA, Gui-shan GU, Cheng-xue WANG, Dong ZHU, Xi-zheng ZHANG. Relationship between surgical time and postoperative complications in senile patients with hip fractures. Chin J Traumatol 2010;13:167-72

8. Martínez A. Fracturas de cadera en ancianos. Pronóstico, epidemiología. Aspectos generales. Experiencia. Rev Col de Or Tra. 2005;19:21-28.

9. Uribe A, Castaño DA, García AN, Pardo EE. Morbilidad y mortalidad en pacientes mayores de 60 años con fractura de cadera en el Hospital Universitario San Vicente Fundación, Medellín, Colombia. Iatreia 2012;25:305-13.

10. Henderson CY, Ryan JP. Predicting mortality following hip fracture: an analysis of comorbidities and complications. Ir J Med Sci. 2015;184:667-71. doi: 10.1007/s11845-015-1271-z

11. Ridgeway S, Wilson J, Charlet A, Kafatos G, Pearson A, Coello R. Infection of the surgical site after arthroplasty of the hip. J Bone Joint Surg Br 2005;87:844-50.

12. Keene GS, Parker MS, Pryor GA. Mortality and morbidity after hip fracture. BMJ 1993;307:1248-50.

13. Negrete J, Alvarado JC, Reyes S. Fractura de cadera como factor de riesgo en la mortalidad en pacientes mayores de 65 años. Estudio de casos y controles. Acta Ortopédica Mexicana 2014;28:352-62.

14. Menéndez ME, Neuhaus V, Ring D. Inpatient mortality after orthopaedic surgery. Int Orthop 2015;1307-14.

15. Alarcón T, González JI. Fractura osteoporótica de cadera. Factores predictivos de recuperación funcional a corto y largo plazo. An. Med. Interna 2004;21:87-9

16. Berrío MI. Envejecimiento de la población: un reto para la salud pública. Rev Colomb Anestesiol 2012;40:192-4.

17. Ruiz ED, Arrubla DJ, Sanabria P. Envejecimiento y vejez en Colombia. Estudio a profundidad. Profamilia. 2013.

18. Colombia. Ministerio de Salud y Protección Social. Sistema nacional de estudios y encuestas poblacionales para la salud. Encuesta de salud, bienestar y envejecimiento - SABE. Bogotá; 2013. 
19. Pontificia Universidad Javeriana. Instituto de Envejecimiento. Bogotá. Experiencias a partir de la encuesta SABE. 2013.

20. García J, Guerrero EA, Terront A, Molina JF, Pérez C, Jannaut MJ, et al. Costos de fracturas en mujeres con osteoporosis en Colombia. Acta Med Colomb 2014; 39:46-56.

21. Rodríguez-Ocampo MV, Betancourt-Urrutia VF, Montoya-Rojas JP, Bautista-Botton DC. Sistemas y modelos de salud, su incidencia en las redes integradas de servicios de salud. Rev. Gerenc. Polit. Salud. 2013;12(24):114-29.

22. Colombia. Ministerio de la Protección Social. Política nacional de envejecimiento y vejez. Bogotá; 2007.

23. Secretaría de Salud de Medellín (SSM) / NACER Salud Sexual y Reproductiva. Percepción de los usuarios y los agentes de salud de la calidad de los servicios de protección específica y detección temprana, en la ciudad de Medellín 2010-2011. Secretaría de Salud de Medellín (SSM); 2012.

24. Young Y, Resnick B. Don't worry and be positive: What helps the most in functional recovery ones year after hip fracture? An exit interview. Rehabil Nurs 2009;34(3):110-17. doi: 10.1016/j.berh.2014.01.001

25. Griffiths F, Mason V, Boardman F, Dennick K, Haywood K, Achten J, et al. Evaluating recovery following hip fracture: a qualitative interview study of what is important to patients. BMJ Open2015;4:e005406. doi:10.1136/bmjopen-2014-005406

26. Al-Busaidi ZQ. Qualitative research and its uses in health care. SQUMJ 2008;8:11-9.

27. Lotus Shyu YI, Chen MC, Liang J, Wu CC, Su JY. Predictor of functional recovery for hip fractured elders during 12 months following hospital discharge: a prospective study on a Taiwanese sample. Osteoporos Int 2004;15:475-82.

28. Lotus Shyu YI, Chen ML, Chen MC, Wu CC, Su JY. Postoperative pain and its impact on quality of life for hip fractured older people over 12 months after hospital discharge. J Clin Nurs 2009;18:755-64. doi: 10.1111/j.1365-2702.2008.02611.x 